\title{
Eine Kongruenz-Erscheinung im Wogulischen
}

Die Kongruenz des Subjekts mit dem Prädikat im Numerus ist eine typische Eigentümlichkeit vieler Sprachen, unter anderem auch der indoeuropäischen und finnisch-ugrischen. Wenn das Subjekt also in der Einzahl steht, wird das Prädikat ebenfalls im Singular auftreten, und sollte das Subjekt in der Mehrzahl stehen, so erscheint auch das Prädikat im Plural. Symbolisch ausgedrückt:

$$
\mathrm{S}_{\mathrm{sg}}-\mathrm{P}_{\mathrm{sg}} \quad \mathrm{S}_{\mathrm{pl}}-\mathrm{P}_{\mathrm{pl}}
$$

Einige Beispiele aus den indoeuropäischen Sprachen: dt. das Kind spielt - die Kinder spielen, fr. l'enfant joue -- les enfants jouent, engl. the child plays - the children play, russ. ребенок играет - реблта играют.

Aus den finnisch-ugrischen Sprachen: fi. lapsi leikkii - lapset leikkivät, estn. laps mängib - lapsed mängivad, syrjV kaga

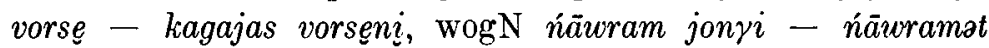
jonyerat, ung. a gyermek játszik - a gyermekek játszanak.

Im Falle eines nominalen Prädikats ist dieselbe Regel gültig: russ. эта птиџа красная - эти птиџы красные, ung. еz а madár piros - ezek a madarak pirosak, wogN $t i$ üjrośs kēlp - $t i$

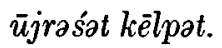

Dieselbe Regel gilt (ausser in Wogulischen) auch in dem Fall, wenn das Subjekt ein Substantiv mit kollektiver Bedeutung ist: dt. das Heer nähert sich, fr. l'armée s'approche, engl. the army nears, russ. ар.мия приближсается; fi. sotajoukko lähestyy, estn. söjavägi läheneb, ung. a sereg közeleg közeledik.

Neben einigen Substantiven mit kollektiver Bedeutung ist eine $\mathrm{g} \mathrm{r}$ a m m at is che Kongruenz, also die Einzahl, im Wogulischen ebenfalls möglich: mãxam lakwi das Volk nähert sich; aber die log i s c h e Kongruenz, also die Mehrzahlform ist jedoch viel häufiger: mãxam lakweyat. Während also in den 
anderen erwähnten Sprachen die oben genannte Regel auch im Fall eines Subjekts mit kollektiver Bedeutung gültig ist: $\mathrm{S}_{\mathrm{NCsg}}-\mathrm{P}_{\mathrm{sg}}$, erscheint im Wogulischen folgende Form: $\mathrm{S}_{\mathrm{NCsg}}-$ Ppl.

Diese Erscheinung war schon früher gut bekannt. Sie wird auch von Kannisto erwähnt: "Das Prädikat steht im Plural, weil das seiner Form nach singularische Subjekt kollektiv ist” (WVd. I 409). Eine ausführliche wogulische Satzlehre ist aber bisher noch nicht erschienen, wir können nur den erwähnten ähnliche, spärliche Äusserungen finden, aus denen noch nicht eindeutig klar wird, was für Kollektiva es sein mögen, die in Subjektfunktion das Prädikat im Plural regieren. Wir können aus ihnen weder auf die mundartliche Streuung und Verbreitung der Erscheinung, noch darauf folgern, neben welchen Kollektiva auch eine grammatische Kongruenz möglich ist. Um auf diese Fragen eine Antwort geben zu können, habe ich die vier Bände von Munkácsis "Vogul Népköltési Gyüjtemény", teils sein Wortmaterial in Handschrift, die sechs Bände der Wogulischen Volksdichtung (Kannisto-Liimola) untersucht, weiterhin meine zur Herausgabe vorbereiteten Texte, die ich 1957/8 und 1966 gesammelt habe. Obwohl die Sammlung von Munkácsi aus den Jahren 1888/9, die von Kannisto aus d.J. 1901/6 stammen und meine Texte dagegen ganz rezent sind, hat sich der Sprachgebrauch in dieser Hinsicht nicht verändert.

Ich habe insgesamt 202 Belege, 161 davon weisen die logische Kongruenz auf (das Prädikat steht im Plural) - $81 \%-, 41$ die grammatische (Prädikat im Singular) - $19 \%$.

Da nun keine lautliche sondern nur eine syntaktische Frage geklärt werden soll, gebe ich meine Beispiele in einer einheitlichen und vereinfachten Transkription an. Die Beispielsätze werden abgekürzt, als Subjekt-Prädikat-Syntagmen angegeben, in allen Fällen, wo die weiteren Satzteile hinsichtlich der Kongruenz von keiner Bedeutung sind. Die einzelnen Mundarten sind natürlich nicht gleichmässig vertreten, aus den nördlichen und östlichen Mundarten stehen uns ja wesentlich mehr Texte zur Verfügung als aus den westlichen und südlichen. Von den parallelen Texten Kannistos aus dem Konda-Gebiet gebe ich die Beispiele des Textes A an, ich weise auf den Text B 
nur hin, wenn dort bezüglich der Kongruenz eine andere Lösung zu finden ist.

Die hierzu gehörenden Wörter (Kollektiva) sind folgende:

N $m \bar{a} \chi \partial m \sim m \bar{a} m$, LM LU V mèm, P mèkam 'Volk, Leute' (Prädikat im Plural): N māxam lāweyat die Leute sagen (VNGy.

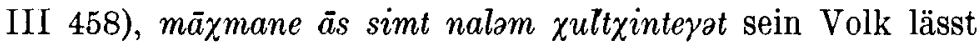
sich da unten in der Mitte des Ob (im Boot) schaukeln (ib. II

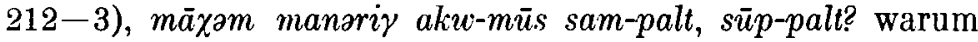
hat das Gesindel nur ein Auge und einen krummen Mund? (ib. I 26), jīwtil alyal māxam òlmat am Oberlauf des Ivdel lebten Menschen (WVd. I 102), āsmāxam ōleyat die Leute am Ob leben (für sich hin) ... ätasłlaxtasat māxam ti minmirtasat die Leute rüsteten sich (und) machten sich nun auf (ib. 194); LM mèm tank kańsian das Volk soll es wissen (VNGy. IV 416); LU mẹm lült die Leute sind schlecht (Munk. Mskr.); V pè l kèt mẹmm kujēt im Dorfe schlafen die Leute (WVd. IV 336); P mẹkam ti kuolēst die Leute gingen zu Ende (ib. II 183), mēkam joxtēt Menschen kommen (ib. III 146). Weitere Beispiele: N VNGy. I 8, 114-5, II 129, 429, III 229, 342-3, IV 149, 410, Munk. Mskr. zwei Beispiele, Kálmán Mskr. neun Beispiele, WVd. I 195, 204, 217, 218, II 139, 168, III 45, V 40, 101, 115, 233; LM Munk. Mskr. ein Beispiel; P WVd. III 146, 147. Prädikat im Singular: N mām rawpi das Volk stirbt aus (VNGy. II 216-7); LU mēm näüriś repi das Volk fällt (stirbt aus) sehr (schnell); P män-šawt mè kam joxtas? wieviele Leute sind gekommen? (die letzteren zwei Beispiele stammen aus Munkácsis Manuskript). - Insgesamt haben wir also 42 Belege für die logische und 3 für die grammatische Kongruenz.

$\mathrm{N}$ mir, K mar 'Volk, Leute' (Prädikat im Plural): N mir kwona-kuāleyat das Volk kommt heraus (VNGy. I 9-10), mir zujasat das Volk hat sich niedergelegt (ib. IV 34), miram usnuwat mein Volk wäre zugrunde gegangen (ib. 37), mir punk pinsat das Volk neigte den Kopf (WVd. I 64), mirmen wāytal patmat unsere Leute wurden müde (ib. 71); K mar löatkatat die Leute sprechen untereinander (ib. II 41). Weitere Beispiele: X VNGy. I 8, 9, 25, 68, 72, 74, 75 (2), 158, II 60, 167, 306, 308, 
IV 234,410, WVd. I 20, 64, 70 (2), 81, 213, 217, 229, II 62, 63, $64,69,124,125$ (2), $126(2), 159,170,171$ (3), 172, III 15, 33 (2), IV 36, 38, 545-6, V 52, VI 144. - Gegenbeispiele (Prädikat im Singular): N mir tēlas die Menschheit kam zustande (VNGy. I 154), mir nänki Leute sind zu sehen (WVd. II 86). Weitere Beispiele: VNGy. I 9, 74, 158, II 12, IV 142, 333, 410, WVd. II 87,173 . - Insgesamt 48 Fälle für die logische und 10 für die grammatische Kongruenz.

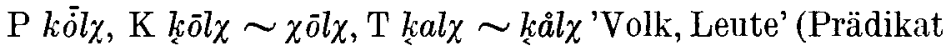
im Plural): P kōl $\chi$ worna ti sìjest die Leute entflohen(?) nun in den Wald (WVd. IV 243); K ḩōl $\chi$ loåttat die Leute sagen (VNGy.

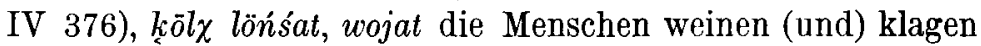
(WVd. I60), $\chi \bar{o} l \chi \bar{a} j \chi w$ pätsat die Leute begannen zu trinken (ib. II

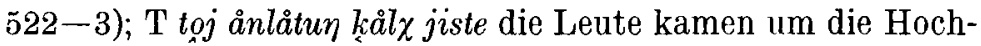

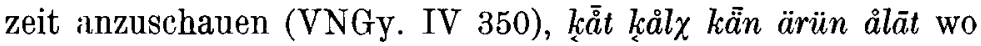

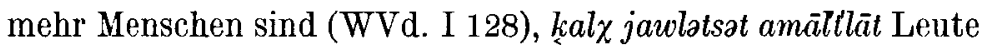
versammelten sich, sie sprechen (jb. III 162). Weitere Beispiele: P Munk. Mskr.; K VNGy. II 239, IV 378 (2), Munk. Mskr., WVd. I 60, II 26, 304-5 (2), 326-7, 512-3, 522-3, 524-5, 526-7, 576--7, 639, 642, V 126; T Munk. Mskr. -

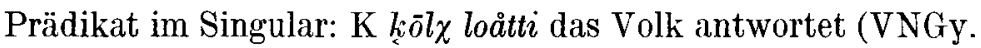
IV 376), $\chi \bar{o} l \chi$ päri-manəst (Pl.), tūls (Sing.) das Volk ging zurück, entfloh (jedoch im Text B tülsat, also im Plural; WVd. II $526-7)$. - Insgesamt 29 Fälle mit logischer und 2 mit grammatischer Kongruenz.

$\mathrm{N} \chi \bar{o} n t, \mathrm{P}$ kōnt, $\mathrm{K}$ kุōnt 'Heer, Kriegsheer' (Prädikat im Plural) $\mathrm{N} \chi \bar{o}$ nt wotamtawesat das Heer wurde vernichtet (VNGy.

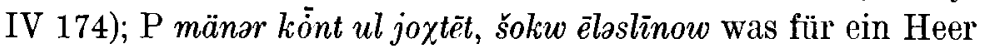
auch kommen mag, wir töten alle (WVd. 182), kónt minēsat das

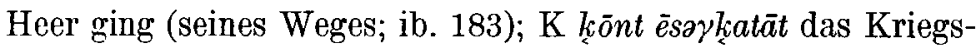

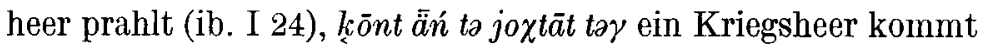
jetzt hierher (ib. II 24). Weitere Beispiele: K WVd. II 31 , 338-9 (3), 649. - Prädikat im Singular: N saran ōtar $\chi \bar{n} n t e$ rō $\chi u w l a ̄ l i$ das Heer des syrjänischen Fürsten schreit (VNGy. I 190); P kónt jalloåli das Heer geht (weiter; WVd. II 6); $\mathrm{K}$ ōnt läkwi die Kriegerschar nähert sich (ib. 422-3). Weitere 
Beispiele: N VNGy. IV 270 (2), WVd. II 49, 159; P ib. 183; $\mathrm{K}$ ib. 24, 43-4, 647 (2), 657. - Insgesamt 10 Fälle für die logische und 13 für die grammatische Kongruenz.

$\mathrm{N} w \bar{a} n t, \mathrm{LM}$ woånt, K wöäont 'Schwarm' (Prädikat im Plural): $\mathrm{N}$ sāt jatrip jatri-wānt tuw issat ein Birkenhuhnschwarm von sieben Birkhühnern hat sich dorthin gesetzt (VNGy. IV 230); LM tẹri-woånt nalam tunśsentēt ein Kranichschwarm steht im

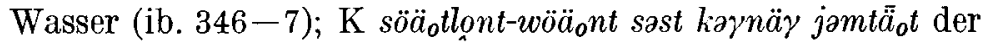
Schwarm von sieben Gänsen wird auf dem Rücken leicht (WVd. III 58). Weitere Beispiele: N VNGy. IV 153, 231, WVd. II 175 (2); LM VNGy. III 346-7 (2), IV 166 (2); K WVd. III 58. - Gegenbeispiele: N lunt-wãnt minenti ein Schwarm von Gänsen geht (dorthin; VNGy. IV 153); LM lunt-woånt minenti id. (ib. 166). Ein weiteres Beispiel: LM ib. III 346-7. - Insgesamt 12 Fälle für die logische und 3 für die grammatische Kongruenz.

K oàtam oåtam 'Volk, Leute' (Prädikat im Plural): oåtam-pal k̨wålilat sogar das Volk geht zu Ende (VNGy. II 231-2), manast jätam juw das Volk ging nach Hause (WVd. I 114). Weitere Beispiele: WVd. I 117, II 10-13, 214-5, 300-1, 472-3, 654 und Munk. Mskr. (2). - Gegenbeispiele: ö̈̈̈tam ōls ein Volk wohnte dort (ib. 10) und 204-5, 304-5, 310-1. Insgesamt 10 Prädikate im Plural, 4 im Singular.

N LM ńūp 'Hochzeitsleute' (Prädikat im Plural): LM äm ńūpäm joxtsat meine Hochzeitsleute sind angekommen (Munk. Mskr.). - Prädikat im Singular: N ńūp joxtenti Hochzeitsleute kommen an (VNGy. IV 270).

Andere Subjekte mit kollektiver Bedentung, Prädikat im

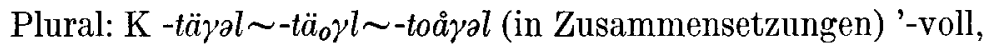
ganz': pềul-täyal, ūs-täyal ķojät das ganze Dorf, die ganze

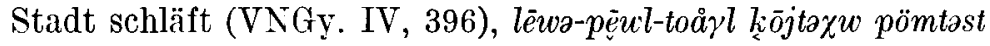
die Bevölkerung des Dorfes Ljeva begann zu siechen (WVd. I

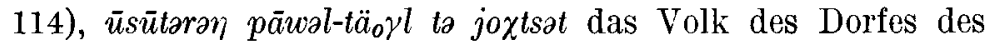
Stadtfürsten kam nun an (ib. II 520-1); $\mathrm{N}$ jēwar 'Schar, 
Rudel, Schwarm': sāt luwpa luw-jēwar tot jōmanteyat eine Schar von sieben Pferden geht dort herum (VNGy. III 334-5); jūtar id.: an luw-jūtar, $\bar{u} j$-jūtar ōs ta ojasat jetzt entfloh auch die Pferdeschar, Rentierschar (ib. 68); LM äñä 'Haufen, Schar, Schwarm': lunt-änä kcanšäm täw luwän tuw täxtəpoåliän der Gänseschwarm überreichte den Brief seinem Pferd (ib. IV 166); P wujkul 'Tier': pot wujkul ètkatsat die Vögel versammelten sich

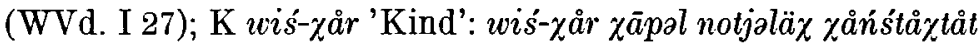
die Kinder (das Kind) lernen, (wie man sich) »in der Länge« (des schnell strömenden Flusses) herunterlassen (soll; Kálmán Mskr.). Insgesamt 9.

Prädikat im Singular: N mujkwe 'Hochzeitsleute': mujkwe joxtantalam Hochzeitsleute sind angekommen (VNGy. IV 270); priliata 'Brigade': zatańewna tomna prikatate rüpiti Chatanjeva Tomnas Brigade arbeitet (Kálmán Mskr.), brigada $\chi \bar{u} l$ aləśli die

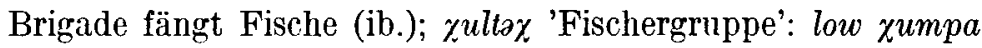

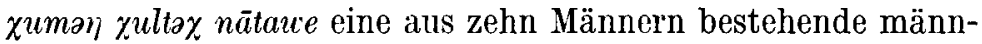
liche Fischergruppe fährt dort im Boot (VNGy. III 498); $\mathrm{K}$ sèxwtal 'Rudel': soåt-wojp sêxutal noålaw wojlas ein Rudel von sieben Elentieren stieg hinab (WVd. I 98). Insgesamt 5 Beispiele.

Auch andere Substantive können eine kollektive Bedeutung haben und als Subjekt sowohl pluralische als auch singularische Prädikate haben, z.B. X' sāli ōśsam, akw pāle zolas ich hatte Rentiere (Sing.), die eine Hälfte von ihnen ging zu Ende (WVd. II 96), ti mā janital ruś ôli, māńśi ōli überall auf dieser Erde sind Russen, sind Wogulen (beide im Sing., ib. 124); K än man jäni losamōl juutaðtsat die Knochen (Sing.) von uns alten Leuten sind jetzt gebrechlich geworden (ib. 436-7).

In der P-Mundart werden einige Substantive mit kollektiver Bedeutung öfters in der Mehrzahl gebraucht und dann stimmen die logische und die grammatische Kongruenz überein, z.B. péul tāyl mìrēt ètkatsat die Leute des ganzen Dorfes versammelten sich (WVd. IV 339). S. noch mirēt 'Leute' (ib. 71) und kólēkt id. (ib. 243).

Zusammenfassend kann folgendes festgestellt werden: im Wogulischen kann das Prädikat neben einem Subjekt mit kollektiver Bedeutung sowohl im Plural (logische Kongruenz) als 


\section{Übersichtstabelle}

Bedeutung

wog. Wort

Prädikat

im Plural im Singular

Volk, Leute

Heer

Brigade

Fischerschar

-voll, ganz

Hochzeitsleute

$\left\{\begin{array}{l}m \bar{a} \chi \partial m \\ m i \dot{ } \\ \chi \bar{o} l \chi \\ \dot{o} \dot{a} t a m\end{array}\right.$

$\chi \bar{o} n t$

prikata

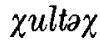

-täyal

$\left\{\begin{array}{l}n \dot{u} p \\ m u j(k w e)\end{array}\right.$

Schar,

Schwarm,

Rudel

Tier

Kind

Insgesamt $\left\{\begin{array}{l}\text { wānt } \\ j \bar{e} w a r \\ j \bar{u} t a r \\ \ddot{a} \bar{n} \ddot{a} \\ \text { sēxwtal }\end{array}\right.$

wujkul

wis'- $\chi a r$
42

48

29

10

10

-

1

4

1

1

- 1

$12 \quad 3$

1

1

1

- 1

1

1

161

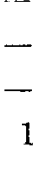

41
2

3

10

2

4

13

2

auch im Singular (grammatische Kongruenz) stehen. Auffallend ist jedoch die Vorherrschaft des pluralischen Prädikats (81\%). Bei Wörtern mit der Bedeutung 'Volk, Leute' kann sie sogar $90 \%$ erreichen.

Mit ähnlichen Verhältnissen können wir auch im nördlichen

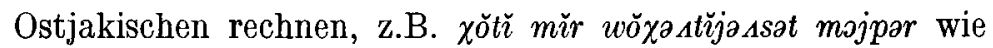
die Leute den Bären erlegt haben (RÉdeI Nordostj. Texte 40-1), isa mir sesat-jañ 'sat das ganze Volk isst und trinkt

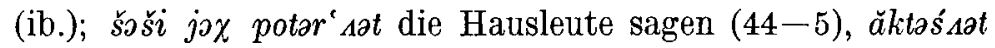
$j o \chi$ die Leute versammelten sich $(46-7)$. Weitere Beispiele im erwähnten Werk: SS. $44-5,46-7,48-51,80-3,112-3$, $114-5,124-5$.

Diese Erscheinung der obugrischen Kongruenz kann auch in den anderen finnisch-ugrischen Sprachen vorkommen. Der berühmt gewordene tscheremissische Satz Regulys: kombo počela 
čongeštat 'die Gänse (Sing.) fliegen hintereinander' kann demnach vielmehr eine ähnliche Konstruktion mit kollektivem Subjekt und mit dem Prädikat im Plural darstellen (ERDődi Mélanges Sauvageot S. 76) als die Überlieferung einer uralten Inkongruenz, wo die Mehrheit nur noch mit dem Prädikat bezeichnet wurde (RAvILA FUF XXVII 96-9).

BÉLA KáLMáN 